\title{
Editorial \\ Social Support: A Relationship Process, Not a Commodity
}

Almost 20 years ago Gerald Caplan (1974), John Cassel (1974), and Sydney Cobb (1976) each wrote highly influential articles on the subject of social support, triggering a barrage of empirical and conceptual investigations of the role of human attachments in the stress and coping process. Each of these scholars emphasized a different aspect of the social support construct. Caplan concentrated on the basic mental health functions of "support systems," suggesting several ways to strengthen their ability to meet people's psychosocial needs, particularly under stressful conditions. Cassel spotlighted the epidemiological significance of social support, citing evidence that social isolation posed a risk to health, and that social integration played a protective role. Cobb's legacy was to provide a definition of social support that emphasized the psychological sense of being loved, cared for, and connected to a reliable network of associates. In addition, he placed this definition of social support in a conceptual framework which is now generally referred to as the stress-buffering model of social support. The model proposes that, under conditions of stress, those people who feel supported in certain ways by particular associates will be insulated from the adverse effects of the stress.

In this issue of the Canadian Journal on Aging Philippe Landreville and Philippe Cappeliez carefully review the literature on the relationship between social support and depressive symptoms among the elderly. They consider the evidence for two different versions of the stress buffering model, one revealing that social support decreases depression through its interaction with stressors (the moderator version) and the other revealing that stressors activate support, which in turn diminishes stress (the mediator or support mobilization version). The authors also contrast this buffering model with the direct effect model which proposes that social support contributes to general well-being, independent of stress. In the context of depression, a significant negative correlation between support and symptomatology constitutes evidence favouring this model.

Landreville and Cappeliez's review leads them to conclude that the weight of the evidence favours both the stress buffering and the direct effects of social support on depression. However, this conclusion is offered with numerous cautions, due to the fact that few studies have tested the two models on the same sample, that studies differ a great deal in the sampling of elderly persons and in the measures of support, and that the majority of studies are cross-sectional rather than longitudinal in design. They also point out that there is confusion about the kinds of measures that reflect the structural dimensions of support and those that gauge its functions. For 
example, they observe that the presence of a confidant has been interpreted by some researchers as a structural indicator of support, and by others as an indicator of the respondent's access to certain supportive provisions.

Landreville and Cappeliez maintain that one reason why the empirical evidence does not consistently favour one model of support over the other is because the studies reviewed do not disaggregate stressors into particular stressful demands faced by the respondents, and fail to examine how different types of support from different providers influence coping and exert an impact on mental health. Accordingly, the authors call for more precision in identifying the kinds of support that are needed in the wake of different life stressors, and the sources of support that are capable of meeting these needs. They believe that more fine-grained analyses will reveal the exact conditions under which social support has a stress buffering effect versus a direct effect on mental health.

I beg to differ. Here are some of the reasons why I disagree with these and other authors who maintain that unresolved questions surrounding the role of social support in the stress process will be answered by studies that discern how particular types and sources of support are matched to the stressful demands aroused by different stressors. First and foremost, we must reconsider the ways in which we conceive and measure social support. When Caplan, Cassel, and Cobb originally introduced their ideas about the concept they were primarily concerned with the health protective functions of people's natural social ties. They speculated that the members of the social network provided feedback about one another's role performance and self-worth, that they shared one another's burdens, and that they provided companionship, advice, emotional support and practical assistance. But the emphasis was always on the capacity of a special social unit to communicate this information and aid. Its special character derived from the significance of the network members to the individual under study. In short, the network's supportive influence stemmed from the significance which the individual assigned to his or her relationship with its members.

When social support became the subject of numerous empirical inquiries, measures were developed which lifted the resources from their proper relationship context. Researchers concentrated exclusively on the provisions relayed by the network, distinguishing between emotional, tangible, and esteem support, and lost sight of the crucial fact that it was the very existence of a prior relationship which brought supportive meaning to interactions. Moreover, when support was removed from relationships, it came to be seen as a commodity that people exchanged rather than one among a large number of interpersonal processes that occur in the conduct of human relationships. Equally important, it gained the status of a variable that could be measured in terms of a fixed quantity and relative quality when it should have been seen as a process that is dynamic and subject to a complex set of contingencies that influence social interaction.

By asking respondents to evaluate the resources they actually receive or those which they believe they could gain from their networks, researchers 
gain no insight into the ways in which social interaction influences health and well-being. Inquiry should focus on the actual stress related transactions that occur with particular network members, not on global evaluations by the respondent of the quantity, quality, or general adequacy of support. Moreover, by concentrating exclusively on the supportive dimension of these transactions, researchers miss the opportunity to identify other interpersonal processes that are likely to shape behaviours, cognitions, and emotions. For example, there is evidence that expressions of conflict, hostility, criticism, or overprotectiveness can neutralize the protective effects of expressions of support. K.S. Rook (1984) found that conflict in relationships was more strongly associated with the well-being of elderly widows than was support. And in the context of depression, I.H. Gotlib and J.M. Hooley's (1988) literature review reveals how measures of multiple interactional processes, not just support, have advanced our understanding of the causal link between depression and marital distress.

When measurement shifts from a narrow focus on the resources people extract from others to the process of interaction in close relationships, the study of social support becomes at once more complicated and more faithful to reality. It is more complicated because people bring different expectations to different relationships, have different stakes in these relationships, and are therefore affected differently by their interactions with different people. Let me illustrate this with an example. In a recent study, M. Steinberg and I (Steinberg \& Gottlieb, forthcoming) interviewed women in dual income marriages about the support they received from their husbands when episodes of conflict occurred between their job and their family demands. After describing how their husbands had become involved in dealing with the conflicts, the women appraised how supportive their husbands had been, using a seven-point scale of helpfulness. However, when we inquired about the reasons for their ratings, we learned that each rating actually reflected a mental averaging of several criteria. For example, a husband who left work to take a sick child home from school received high marks for his contribution to resolving a crisis, but received low marks because his wife had to pressure him into taking this action and because she had to pay for his help in other ways after she returned home from work. In short, a positive impact on the outcome of the crisis was counteracted by the unpleasantness of having to plead for the assistance and by the debt the wife incurred.

This example goes to show that interactions cannot be neatly divided into mutually exclusive supportive and unsupportive categories, but that they have elements of both. It also reveals that relationships colour the supportive meaning of interactions because people bring different expectations to different relationships. Equally important, the diverse processes that occur in different relationships not only exert an impact on the outcomes by altering the trajectory of coping, but also influence the quality and course of the relationship itself. When a wife must solicit her husband's help in an emergency involving their child, and when she must make reparations to 
him in the wake of the crisis, she is likely to feel resentful toward her partner and question the mutuality and commitment in the marriage. Although the crisis may have been resolved, the process of its resolution and its psychological effects may have engendered additional tensions in the relationship.

What are the implications of this perspective for the study of the relationship between social support and depression among the elderly? If empirical studies were to emphasize the interactions that occur in close relationships, fully recognizing the social character of human ties, then we would no longer have to conclude our investigations with the familiar lament that more research is needed on the process or the mechanisms underlying social support's effects on depression or other mental health outcomes. This is because we would conduct more painstaking investigations of the interactions that unfold in the conduct of human relationships, using in-depth interviews, daily diaries, interaction records, and observational coding schemes. These methods would allow us to view social support as a dynamic process that is subject to negotiation and influenced by a host of personal, situational, and relational factors. In the context of depression, these methods would allow us to document how the life stressors and transitions experienced by the elderly are affected by and affect their relationships with key figures in their networks, and how particular interactions affect mood, morale, and the maintenance of relationships in the network.

Support is only one of many messages that might affect the onset and course of depressive affect. Avoidance, withdrawal, anger, pity, and blame are other messages that can be communicated, despite our best intention to be of use to others. Depressive affect and behaviour can be threatening to would-be helpers, and are typically resistant to change in the short term. It is therefore both discouraging and depressing to spend much time with the depressed. Moreover, exposure to a depressed elderly person is likely to arouse even more intense feelings of helplessness and threat due to deeply ingrained cultural beliefs about the vulnerability that comes with old age. Perhaps then the study of support in the context of depression must not only take interpersonal communications into account, but must also consider how the broader cultural script conditions these patterns of communication.

Finally, we may need to remind ourselves that, across the entire lifespan, transient depressive mood is a commonly-experienced affective state, and represents one shading in the broad spectrum of human emotions. There are occasions when it is the fullest and most appropriate expression of our sentiments, such as when it arises in response to social losses. On these occasions, caring and compassion are the best expressions of support, not efforts to sanitize or dispel distress. 


\section{References}

Caplan, G. (1974). Support systems. In G. Caplan (Ed.), Support systems and community mental health. New York: Basic Books.

Cassel, J. (1974). Psychosocial processes and stress. Theoretical formulations. International Journal of Health Services, 4, 471-482.

Cobb, S. (1976). Social support as a moderator of life stress. Psychosomatic Medicine, 38, 300-314.

Gotlib, I.H., \& Hooley, J.M. (1988). Depression and marital distress: Current status and future directions. In S. Duck (Ed.), Handbook of personal relationships (pp. 543-570). New York: John Wiley \& Sons.

Rook, K.S. (1984). The negative side of social interaction: Impact on psychological well-being. Journal of Personality and Social Psychology, 46, 1097-1108.

Steinberg, M., \& Gottlieb, B.H. (forthcoming). Appraisals of spousal support among women facing conflicts between work and family. In B. Burleson, T. Albrecht, \& I. Sarason (Eds.), Communicating social support. Newbury Park, CA: Sage Publications.

Benjamin H. Gottlieb, University of Guelph 